\title{
Non-steroidal anti-inflammatory drugs and risk of heart failure in four European countries: nested case-control study
}

\author{
Andrea Arfè,, Lorenza Scotti, ${ }^{1}$ Cristina Varas-Lorenzo, ${ }^{2}$ Federica Nicotra, ${ }^{1}$ Antonella Zambon, ${ }^{1}$ \\ Bianca Kollhorst, ${ }^{3}$ Tania Schink, ${ }^{3}$ Edeltraut Garbe, ${ }^{3}$ Ron Herings, ${ }^{4}$ Huub Straatman, ${ }^{4}$ René Schade, ${ }^{5}$ \\ Marco Villa, ${ }^{6}$ Silvia Lucchi, ${ }^{6}$ Vera Valkhoff, ${ }^{5}$ Silvana Romio, ${ }^{5}$ Frantz Thiessard, ${ }^{7}$ Martijn Schuemie, ${ }^{5}$ \\ Antoine Pariente, ${ }^{7}$ Miriam Sturkenboom, ${ }^{5}$ Giovanni Corrao ${ }^{1}$ On behalf of the Safety of Non-steroidal \\ Anti-inflammatory Drugs (SOS) Project Consortium
}

1 Unit of Biostatistics,

Epidemiology, and Public

Health, Department of Statistics

and Quantitative Methods,

University of Milano-Bicocca,

20126 Milan, Italy

${ }^{2}$ RTI Health Solutions,

Barcelona, Spain

3Leibniz Institute of Prevention

Research and Epidemiology,

Bremen, Germany

4PHARMO Institute, Utrecht,

Netherlands

${ }^{5}$ Department of Medical Informatics, Erasmus University

Medical Centre, Rotterdam,

Netherlands

${ }^{6}$ Local Health Authority ASL

Cremona, Cremona, Italy

7 University of Bordeaux

Segalen, Bordeaux, France

Correspondence to: G Corrao giovanni.corrao@unimib.it

Additional material is published online only. To view please visit

the journal online.

Cite this as: BMJ 2016;354:i4857 http://dx.doi.org/10.1136/bmj.i4857

Accepted: 01 September 2016

\section{ABSTRACT}

\section{OBJECTIVES}

To investigate the cardiovascular safety of nonsteroidal anti-inflammatory drugs (NSAIDs) and estimate the risk of hospital admission for heart failure with use of individual NSAIDs.

DESIGN

Nested case-control study.

SETTING

Five population based healthcare databases from four European countries (the Netherlands, Italy, Germany, and the United Kingdom).

\section{PARTICIPANTS}

Adult individuals (age $\geq 18$ years) who started NSAID treatment in 2000-10. Overall, 92163 hospital admissions for heart failure were identified and matched with 8246403 controls (matched via risk set sampling according to age, sex, year of cohort entry).

\section{MAIN OUTCOME MEASURE}

Association between risk of hospital admission for heart failure and use of 27 individual NSAIDs, including 23 traditional NSAIDs and four selective COX 2 inhibitors. Associations were assessed by multivariable conditional logistic regression models. The dose-response relation between NSAID use and heart failure risk was also assessed.

RESULTS

Current use of any NSAID (use in preceding 14 days) was found to be associated with a $19 \%$ increase of risk

\section{WHAT IS ALREADY KNOWN ON THIS TOPIC}

Several randomised clinical trials and observational studies have shown an association between use of non-steroidal anti-inflammatory drugs (NSAIDs) and increased risk of heart failure, but the risk and dose-response relation associated with individual NSAIDs is largely unknown

Heart failure was included as an outcome of interest in the Safety of Non-Steroidal Anti-Inflammatory Project, a multinational project funded by the European Commission under the seventh Framework Programme

\section{WHAT THIS STUDY ADDS}

Use of seven individual traditional NSAIDs (diclofenac, ibuprofen, indomethacin, ketorolac, naproxen, nimesulide, and piroxicam) and two individual COX 2 selective NSAIDs (etoricoxib and rofecoxib) is associated with and increased risk of hospital admission for heart failure

Risk of admission for heart failure is doubled for some NSAIDs used at very high doses Estimates of the risk of heart failure associated with the use of many individual NSAIDs in this study could help to inform both clinicians and health regulators of hospital admission for heart failure (adjusted odds ratio 1.19; $95 \%$ confidence interval 1.17 to 1.22 ), compared with past use of any NSAIDs (use $>183$ days in the past). Risk of admission for heart failure increased for seven traditional NSAIDs (diclofenac, ibuprofen, indomethacin, ketorolac, naproxen, nimesulide, and piroxicam) and two COX 2 inhibitors (etoricoxib and rofecoxib). Odds ratios ranged from 1.16 (95\% confidence interval 1.07 to 1.27 ) for naproxen to 1.83 (1.66 to 2.02) for ketorolac. Risk of heart failure doubled for diclofenac, etoricoxib, indomethacin, piroxicam, and rofecoxib used at very high doses ( $\geq 2$ defined daily dose equivalents), although some confidence intervals were wide. Even medium doses (0.9-1.2 defined daily dose equivalents) of indomethacin and etoricoxib were associated with increased risk. There was no evidence that celecoxib increased the risk of admission for heart failure at commonly used doses.

\section{CONCLUSIONS}

The risk of hospital admission for heart failure associated with current use of NSAIDs appears to vary between individual NSAIDs, and this effect is dose dependent. This risk is associated with the use of a large number of individual NSAIDs reported by this study, which could help to inform both clinicians and health regulators.

\section{Introduction}

Non-steroidal anti-inflammatory drugs (NSAIDs) are a broad class of agents with analgesic and anti-inflammatory properties that inhibit the two recognised isoenzymes of prostaglandin $\mathrm{G} / \mathrm{H}$ synthase (also known as cyclo-oxygenase (COX))-namely, COX 1 and COX $2 .{ }^{1}$ Because the therapeutic action of these drugs is mostly mediated by inhibition of $\operatorname{COX} 2$, while their gastrointestinal adverse reactions are largely due to $\mathrm{COX} 1 \mathrm{inhi}$ bition, NSAIDs selectively inhibiting COX 2 were developed in the 1990s to reduce the risk of gastrointestinal toxicity. $^{2}$

Nevertheless, reports of cardiovascular adverse reactions began to emerge in 2000-03, ${ }^{34}$ and subsequent placebo controlled trials showed that COX 2 inhibitors were associated with an increased risk of atherothrombotic vascular events. ${ }^{56}$ However, meta-analyses of randomised trials and observational studies have since shown that the higher cardiovascular risk is not restricted to COX 2 inhibitors, but also applies to some traditional NSAIDs. ${ }^{7-12}$ 
In particular, NSAID use has been found to be associated with an increased risk of heart failure in several randomised clinical trials ${ }^{11}$ and observational studies. ${ }^{1314}$ A large meta-analysis of over 600 randomised trials showed that COX 2 inhibitors and high doses of traditional NSAIDs (that is, diclofenac, ibuprofen, and naproxen) increased the risk of hospital admission for heart failure from 1.9-fold to 2.5-fold compared with placebo. ${ }^{11}$ In the light of this evidence, current guidelines limit the use of NSAIDs in patients predisposed to heart failure, with a full contraindication for patients with diagnosed heart failure. ${ }^{15}$

Nevertheless, there is still limited information on the risk of heart failure associated with the use of individual NSAIDs (both COX 2 inhibitors and traditional NSAIDs) in clinical practice, and especially on their dose-response associations. Therefore, heart failure was included as an outcome of interest in the overall cardiovascular and gastrointestinal risk evaluation of individual NSAIDs within the Safety of Non-Steroidal Anti-Inflammatory (SOS) Project, a multinational project funded by the European Commission under the seventh Framework Programme. A large, common protocol, nested case-control study based on electronic healthcare databases from four European countries was carried out.

\section{Methods}

\section{Data sources}

This study was based on five electronic health databases from four European countries: the Netherlands, Italy, Germany, and the UK. Overall, these databases covered over 37 million people with different time windows of data availability between 1999 and 2010; table 1 summarises their main characteristics.

Briefly, PHARMO is a population based, medical record linkage system covering more than two million inhabitants from the Netherlands. SISR is an electronic administrative healthcare database in Italy, covering the about 10 million residents in the Lombardy region, who all receive free healthcare assistance from the Italian national health service. OSSIFF is a healthcare database covering about three million individuals who are beneficiaries of eight local health authorities in the Lombardy region. Because OSSIFF covers a subset of the population already covered by SISR, we included only the seven million beneficiaries of the Italian national health service not already included in OSSIFF in this study. GePaRD is a claims database in Germany covering about 14 million individuals enrolled in four German statutory health insurance providers. Lastly, THIN is a general practice database comprising primary care medical records from more than 10 million individuals in the UK.

Each database longitudinally recorded data on each member of its target population, including demographic data, hospital discharge diagnoses, and outpatient drug prescriptions. Data on outpatient diagnoses were also available from GePaRD. In two databases (PHARMO and THIN), the daily dose prescribed by physicians was recorded for each dispensed prescription. Further details are reported elsewhere. ${ }^{17}$

\section{Harmonisation and data processing}

Databases differed in several aspects, including type of collected information (that is, healthcare use, claims, and primary care data) and classification systems used for disease and medication coding (table 1). As a result, we performed data harmonisation according to a procedure developed and assessed in the European Union (EU)-ADR (exploring and understanding adverse drug reactions by integrative mining of clinical records and biomedical knowledge) Project ${ }^{18}$ and also implemented in other EU funded projects. ${ }^{19}$ Specifically, the Unified Medical Language system (for clinical diagnoses and conditions) and the Anatomic Therapeutic Chemical (ATC) classification system (for drug prescriptions) were mapped into the coding systems used by the individual databases. This mapping ensured that the data extraction processes targeted the same semantic concepts across all databases, thus allowing analyses to be performed under a common data model. ${ }^{19}$

Anonymised data were extracted locally and processed with Jerboa software (developed by Erasmus $\mathrm{MC}$ ), providing individual level datasets in a common data format. These datasets were securely transferred into the SOS data warehouse, hosted by the University of Milano-Bicocca, to be analysed centrally and securely. ${ }^{19}$

\begin{tabular}{|c|c|c|c|c|c|c|}
\hline Country & Database* & Type of database & $\begin{array}{l}\text { Size of covered } \\
\text { population }\end{array}$ & $\begin{array}{l}\text { Covered } \\
\text { period }\end{array}$ & $\begin{array}{l}\text { Diagnoses } \\
\text { coding }\end{array}$ & Drugs coding \\
\hline Netherlands & $\begin{array}{l}\text { PHARMO (PHARMO Institute for Drug } \\
\text { Outcomes Research) }\end{array}$ & Record linkage & 2.2 million & $1999-2008$ & ICD-9-CM & $\begin{array}{l}\text { Anatomical Therapeutic Chemical } \\
\text { classification system }\end{array}$ \\
\hline \multirow[t]{2}{*}{ Italy } & SISR (Sistema Informativo Sanitario Regionale)† & Healthcare use & 7.5 million & $2003-08$ & ICD-9-CM & $\begin{array}{l}\text { Anatomical Therapeutic Chemical } \\
\text { classification system }\end{array}$ \\
\hline & $\begin{array}{l}\text { OSSIFF (Osservatorio Interaziendale per la } \\
\text { Farmacoepidemiologia e la Farmacoeconomia) }\end{array}$ & Healthcare use & 2.9 million & $2000-08$ & ICD-9-CM & $\begin{array}{l}\text { Anatomical Therapeutic Chemical } \\
\text { classification system }\end{array}$ \\
\hline Germany & $\begin{array}{l}\text { GePaRD (German Pharmacoepidemiological } \\
\text { Research Database) }\end{array}$ & Claims & 13.7 million & 2004-09 & ICD-10-GM & $\begin{array}{l}\text { Anatomical Therapeutic Chemical } \\
\text { classification system }\end{array}$ \\
\hline UK & THIN (The Health Improvement Network) & General practice & 11.1 million & $1999-2010$ & READ version 2 & BNF/Multilex codes \\
\hline
\end{tabular}




\section{Cohort selection and follow-up}

Following the new users paradigm, ${ }^{16}$ a cohort of individuals starting NSAID treatment was selected from all databases. In detail, adults (age $\geq 18$ years) who received at least one NSAID prescription or dispensation (ATC code M01A; excluding topical NSAIDs) during 2000-10 were considered eligible to enter the cohort. The date of first recorded prescription or dispensation was defined as the date of cohort entry. We excluded participants if they:

- Did not have at least one year of uninterrupted observation before the date of cohort entry, to ensure enough time of observation for assessing baseline covariates and applying the next exclusion criteria

- Received one or more NSAIDs within the year preceding the date of cohort entry, to exclude prevalent NSAIDs users

- Received a diagnosis of malignant cancer, with the exception of non-melanoma skin cancers, to exclude patients who may have had particular contraindications

- Were admitted to hospital with a primary diagnosis of heart failure in the year before the date of cohort entry, to avoid the inclusion of events occurring before the start of NSAIDs use (note that secondary hospital or outpatient heart failure diagnoses were not considered as exclusion criteria).

Each cohort member accumulated person years of follow-up, from the date of cohort entry to the earliest date of outcome onset (date of first hospital admission with a primary diagnosis of heart failure), censoring (end of registration in the database due to death or emigration), diagnosis of malignancy (excluding non-melanoma skin cancers), or end of database specific data availability.

\section{Cases and controls}

A case-control study was nested into the cohort of new users of NSAIDs. The endpoint of interest was the first hospital admission for heart failure (that is, with heart failure as the main cause or reason of hospital admission) identified during follow-up. Heart failure is a clinical syndrome involving several pathophysiological mechanisms that, along with factors triggering circulatory decompensation, could produce heterogeneous clinical manifestations that often receive delayed diagnosis. Therefore, our endpoint definition did not include diagnostic codes for clinical heart failure in the outpatient setting and secondary hospital discharge codes for heart failure (which are likely to represent heart failure manifestations occurring during hospital admission for other causes).

Consequently, cases were all cohort members admitted for heart failure during follow-up, identified either from primary hospital discharge diagnoses (PHARMO, SISR, OSSIFF, GePaRD) or codes registered by the general practitioner (THIN). We defined the date of the first admission for heart failure identified during follow-up as the index date. Codes used to identify heart failure cases in each database are reported in the supplementary material (table S1).
We matched each case to up to 100 controls. Controls were randomly selected by risk set sampling from all cohort members whose follow-up did not end before the index date of the considered case (that is, among individuals still at risk of an admission for heart failure). Matching was performed within each database according to sex, age at cohort entry (within 1 year's difference), and date of cohort entry (within 28 days' difference).

\section{Exposure to NSAIDs}

All NSAIDs dispensations received by cohort members during follow-up were identified; this included 27 individual NSAIDs (23 traditional NSAIDs and four selective COX 2 inhibitors). For each cohort member, we directly calculated the period covered by the availability of each individual NSAID by the prescribed daily dose, if available (that is, PHARMO and THIN databases), or by dividing the total amount of drug prescribed for the defined daily dose.

We classified cohort members into the following categories of NSAID use: current, recent, and past. Current users were patients with NSAID availability at the index date or the preceding 14 days. The remaining patients were defined recent users if they had NSAID availability during the time window of 15-183 days before the index date, or past users otherwise (reference).

\section{Covariates}

We assessed several covariates for each cohort member if available in the corresponding database, including:

- History of outpatient or secondary inpatient diagnoses of heart failure, comorbidities, and lifestyle features or clinical characteristics, assessed in the 12 months before cohort entry

- Concomitant use of specific drugs, assessed in the 90 days before the index date.

Comorbidities were assessed by hospital discharge diagnoses (PHARMO, GePaRD, SISR, OSSIFF), outpatient clinical diagnoses (GePaRD), clinical electronic general practice records (THIN), and use of specific drugs. Table 2 reports the full list of covariates.

\section{Statistical analysis}

Individual level data from all databases were firstly gathered into a pooled dataset and analysed by means of a multivariable conditional logistic regression model. ${ }^{20}$ The obtained odds ratio, with $95 \%$ confidence intervals, estimated the risk of hospital admission for heart failure associated with current use of individual NSAIDs with respect to past use of any NSAID. We also estimated the odds ratio associated with recent use of any NSAID, compared with past use of any NSAID. Given the substantial number of associations assessed in this analysis, we used the Bonferroni-Holm procedure $^{21}$ to assess the impact of uncertainty due to multiple comparisons on the results. ${ }^{22}$ Some evidence has supported the relative cardiovascular safety of celecoxib by comparison with other NSAIDs is available in the literature. ${ }^{1423-28}$ Therefore, as a secondary analysis, we estimated the odds ratios measuring the association 


\begin{tabular}{|c|c|c|}
\hline & Case patients $(n=92163)$ & Controls $(n=8246403)$ \\
\hline Men & $41652(45.2)$ & $3671565(44.5)$ \\
\hline Age at cohort entry (years, mean (standard deviation)) & $77(11)$ & $76(10)$ \\
\hline \multicolumn{3}{|l|}{ Comorbidities and other characteristics $^{\star}$} \\
\hline Acute myocardial infarctiont & $3063(3.3)$ & $81222(1.0)$ \\
\hline Alcohol abuse (ATC code starting with N07BB) & $1942(2.1)$ & $128871(1.6)$ \\
\hline Asthmat & $1031(1.1)$ & $57079(0.7)$ \\
\hline Atrial fibrillation and fluttert & $4606(5.0)$ & $110217(1.3)$ \\
\hline Chronic liver diseaset & $1815(2.0)$ & $98762(1.2)$ \\
\hline Chronic respiratory diseaset (ATC code starting with R03) & $16190(17.6)$ & $870497(10.6)$ \\
\hline Diabetest (ATC code starting with A10) & $17888(19.4)$ & $725320(8.8)$ \\
\hline Heart failuret (ATC code C07AG02) & $8353(9.1)$ & $209125(2.5)$ \\
\hline Hyperlipidaemiat (ATC code starting with C10) & $18793(20.4)$ & $1160532(14.1)$ \\
\hline Hypertensiont & $19905(21.6)$ & $1515002(18.4)$ \\
\hline Iron deficiency anaemia (ATC code starting with B03A) & $2159(2.3)$ & $83926(1.0)$ \\
\hline Ischaemic heart diseaset & $8406(9.1)$ & $294986(3.6)$ \\
\hline Kidney failure & $1445(1.6)$ & $41094(0.5)$ \\
\hline Obesity (ATC code starting with A08A) & $4555(4.9)$ & $181104(2.2)$ \\
\hline Osteoarthritis $\dagger$ & $6916(7.5)$ & $483721(5.9)$ \\
\hline Other cardiovascular disease (ATC code starting with C01B) †‡ & $13055(14.2)$ & $463797(5.6)$ \\
\hline Rheumatoid arthritis and inflammatory polyarthritist (ATC code starting with M01C) & $736(0.8)$ & $40269(0.5)$ \\
\hline Smoking & $164(0.2)$ & $8155(0.1)$ \\
\hline Stroket & $1869(2.0)$ & $85109(1.0)$ \\
\hline Valvular disease and endocarditis $\dagger$ & $2383(2.6)$ & $70646(0.9)$ \\
\hline \multicolumn{3}{|l|}{ Concomitant use of other drugs§ } \\
\hline ACE inhibitor/angiotension II antagonistst & $38834(42.1)$ & $2030050(24.6)$ \\
\hline Anticoagulantst & $17589(19.1)$ & $442725(5.4)$ \\
\hline Aspirint & $31658(34.4)$ & $1669443(20.2)$ \\
\hline$\beta$ blockerst & $22506(24.4)$ & $1253749(15.2)$ \\
\hline Calcium channel blockers $\dagger$ & $28911(31.4)$ & $1754965(21.3)$ \\
\hline Cardiac glycosidest & $14429(15.7)$ & $342042(4.1)$ \\
\hline Cyp2C9 inducers & 38 & 1149 \\
\hline Сyp2C9 inhibitors & $8289(9.0)$ & $174253(2.1)$ \\
\hline Diureticst & $48991(53.2)$ & $1536700(18.6)$ \\
\hline Glucocorticoidst & $8636(9.4)$ & $349012(4.2)$ \\
\hline Nitratest & $24029(26.1)$ & $717669(8.7)$ \\
\hline Platelet aggregation inhibitor† & $9105(9.9)$ & $367716(4.5)$ \\
\hline Vasodilatorst & $1654(1.8)$ & $44916(0.5)$ \\
\hline \multicolumn{3}{|c|}{$\begin{array}{l}\text { ATC=Anatomic Therapeutic Chemical (ATC) classification system; ACE=angiotensin converting enzyme; Cyp2C9=cytochrome P450 } 2 \text { C9. } \\
\text { *Comorbidities assessed during the } 12 \text { months before cohort entry, on the basis of inpatient diagnoses, outpatient diagnoses (German GePaRD } \\
\text { database only), medical history (UK THIN database only), or selected drug prescriptions belonging to the indicated ATC codes (only for specific } \\
\text { covariates). } \\
\text { tAvailable in all databases. } \\
\text { fOther cardiovascular diseases include: cardiac arrhythmia or conduction disorders and arrest, cardiomyopathies, peripheral arterial diseases, arterial } \\
\text { embolism and thrombosis, myocarditis, and pericarditis. } \\
\text { \$Drug use assessed during the } 14 \text { days preceding the index hospital admission for heart failure. }\end{array}$} \\
\hline
\end{tabular}

between current use of individual NSAIDs and heart failure risk, using current use of celecoxib as reference. Among the covariates mentioned above, those available in all databases (including history of outpatient or secondary inpatient diagnoses of heart failure) entered the model. We did subgroups analyses after stratification for sex and history of heart failure diagnoses.

Because databases differed with respect to covered populations, as well as type and level of detail of available covariates, we evaluated the robustness of the pooled estimates using a meta-analytic approach by means of the following procedure. Firstly, we separately fitted a conditional logistic regression model to estimate the effect of each individual NSAID within each database. To avoid computational issues (that is, model convergence failure due to sparse data), only individual
NSAIDs with at least five exposed cases were considered in the model. The covariates available for all databases were always forced to enter the model, provided they reached at least 5\% prevalence among controls. Other covariates were included, provided they were significantly $(\mathrm{P}<0.05)$ associated with the outcome in a univariate analysis, and selected from a backward procedure ( $\mathrm{P}>0.10$ for removal). Secondly, we used a random effects meta-analytic model ${ }^{29} 30$ to estimate a summary odds ratio (and 95\% confidence interval) across databases for current use of each individual NSAID (provided that a point estimate was available from at least two databases), compared with past use of any NSAID. Heterogeneity between database specific odds ratios was assessed by Cochran's Q and Higgins' $\mathrm{I}^{2}$ statistics. $^{31}$ 


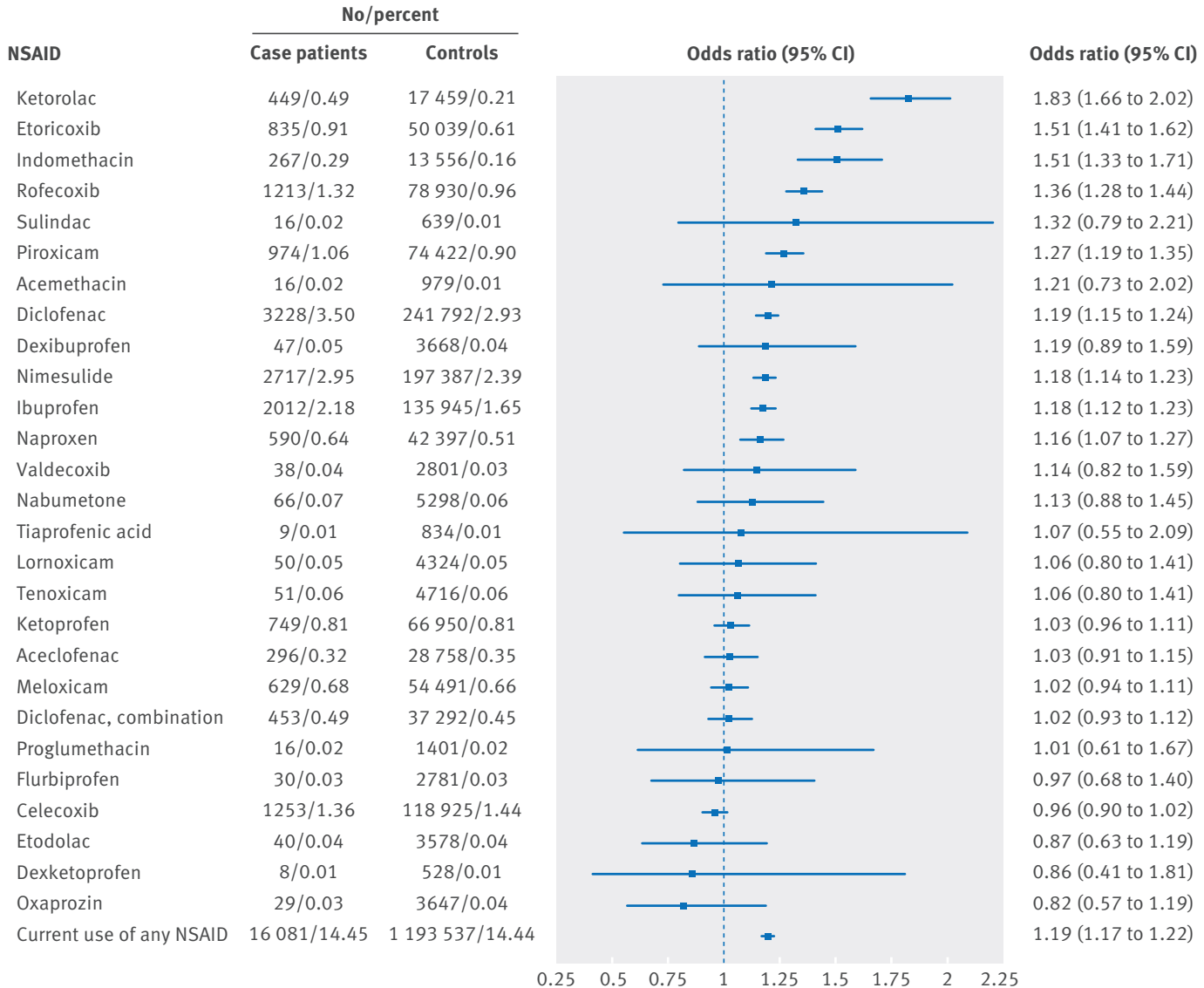

Fig 1 | Distribution of current use of individual NSAIDs among cases and controls and pooled associations between current use of individual NSAIDs and risk of hospital admission for heart failure, with past use of any NSAID as reference. Estimates obtained by pooling individual data from all available databases. Pooled odds ratios and $95 \%$ confidence intervals estimated by fitting a conditional logistic regression model after correcting for available covariates

\section{Dose-response analysis}

We did a dose-response analysis to assess how the risk of hospital admission for heart failure associated with current use of individual NSAIDs varied along the considered categories of prescribed daily dose. Because Italian and German databases did not record data on prescribed daily doses, we pooled individual level data from the Netherlands (PHARMO) and the UK (THIN) databases. Patients for whom the information on the prescribed daily dose was not available were excluded.

The prescribed daily dose was expressed in defined daily dose equivalents (DDD) and categorised as low ( $\leq 0.8 \mathrm{DDD})$, medium (0.9-1.2 DDD), high (1.3-1.9), or very high dose ( $\geq 2 \mathrm{DDD}$ ) with respect to the corresponding defined daily dose. To avoid computational issues, we considered only NSAIDs for which all the considered categories included at least one heart failure case in the analysis. Tests for trends in odds ratios were performed. We did statistical analyses using SAS software (version 9.3; SAS Institute). All tests were two sided and considered significant for $\mathrm{P}$ values less than 0.05 .

\section{Patient involvement}

No patients were involved in setting the research question or the outcome measures, nor were they involved in developing plans for design or implementation of the study. No patients were asked to advise on interpretation or writing up of results. There are no plans to disseminate the results of the research to study participants or the relevant patient community.

\section{Results}

Study cohort

Supplementary figure S1 shows the flowchart describing the attrition of eligible NSAIDs users after exclusion criteria were applied. Among nearly 10 million new users of NSAIDs identified across all databases, 7680181 met the inclusion criteria and constituted the study cohort. Cohort members accumulated 24555063 person years of follow-up and generated 92163 cases of heart failure admitted to hospital (incident rate, 37.5 heart failure events per 10000 person years). Cases were matched to 8246403 controls.

Mean age was 77 (standard deviation 11) years and 76 (10) years among cases and controls, respectively (table 2). About $45 \%$ of both cases and controls were men. Compared with controls, cases had more comorbidities (mainly cardiovascular disease, such as acute myocardial infarction, other ischaemic heart diseases, atrial fibrillation and flutter, and valvular disease and endocarditis) and received concomitant drug treatments more often (eg, anticoagulants, cardiac 


\begin{tabular}{|c|c|c|c|c|c|c|}
\hline & \multicolumn{2}{|c|}{$\begin{array}{l}\text { Risk of admission for heart failure (pooled odds } \\
\text { ratio }(95 \% \mathrm{Cl}) \text { ) }\end{array}$} & \multirow[b]{2}{*}{$P$} & \multicolumn{2}{|c|}{$\begin{array}{l}\text { Risk of admission for heart failure (pooled } \\
\text { odds ratio }(95 \% \mathrm{CI}) \text { ) }\end{array}$} & \multirow[b]{2}{*}{$P$} \\
\hline & $\begin{array}{l}\text { No prior heart } \\
\text { failure }(n=83810)\end{array}$ & $\begin{array}{l}\text { Prior heart failure } \\
(\mathrm{n}=8353)\end{array}$ & & $\operatorname{Men}(n=41652)$ & Women $(n=50511)$ & \\
\hline \multicolumn{7}{|l|}{ Current use of NSAID } \\
\hline Indomethacin & $1.52(1.31$ to 1.77$)$ & $1.58(0.55$ to 4.51$)$ & 0.94 & $1.71(1.41$ to 2.07$)$ & 1.25 (1.00 to 1.57$)$ & 0.04 \\
\hline Sulindac & 1.62 (0.90 to 2.94$)$ & NA & NA & 2.19 (0.80 to 5.97$)$ & 1.50 (0.71 to 3.16$)$ & 0.55 \\
\hline Diclofenac & 1.21 (1.16 to 1.26$)$ & 1.14 (0.91 to 1.42$)$ & 0.61 & 1.21 (1.13 to 1.29$)$ & 1.19 (1.13 to 1.26$)$ & 0.70 \\
\hline Etodolac & 0.83 (0.59 to 1.17$)$ & NA & NA & 0.92 (0.56 to 1.50$)$ & 0.77 (0.48 to 1.23$)$ & 0.61 \\
\hline Acemetacin & 1.67 (0.83 to 3.35$)$ & 0.28 (0.03 to 2.32) & 0.13 & 1.22 (0.52 to 2.85$)$ & 2.08 (0.84 to 5.12 ) & 0.40 \\
\hline Ketorolac & 1.94 (1.71 to 2.19$)$ & 5.09 (0.97 to 26.57) & 0.25 & 1.86 (1.52 to 2.28$)$ & $1.96(1.70$ to 2.27$)$ & 0.68 \\
\hline Aceclofenac & $1.00(0.87$ to 1.14$)$ & 0.89 (0.20 to 3.89) & 0.88 & 1.13 (0.90 to 1.41$)$ & $0.94(0.80$ to 1.11$)$ & 0.19 \\
\hline Diclofenac, combinations & $1.02(0.92$ to 1.14$)$ & 0.89 (0.36 to 2.16$)$ & 0.77 & 1.03 (0.87 to 1.22$)$ & 1.01 (0.89 to 1.16$)$ & 0.86 \\
\hline Piroxicam & $1.31(1.21$ to 1.41$)$ & 1.90 (1.01 to 3.59$)$ & 0.25 & 1.34 (1.18 to 1.53$)$ & 1.31 (1.20 to 1.44$)$ & 0.78 \\
\hline Tenoxicam & 1.03 (0.74 to 1.43$)$ & NA & NA & 0.88 (0.47 to 1.62$)$ & 1.07 (0.75 to 1.53$)$ & 0.59 \\
\hline Lornoxicam & $1.13(0.81$ to 1.57$)$ & 2.25 (0.28 to 18.08$)$ & 0.52 & 1.22 (0.68 to 2.18) & $1.07(0.73$ to 1.56$)$ & 0.71 \\
\hline Meloxicam & 0.99 (0.91 to 1.09$)$ & 0.95 (0.43 to 2.07) & 0.92 & 1.13 (0.97 to 1.31$)$ & 0.95 (0.85 to 1.06$)$ & 0.07 \\
\hline Ibuprofen & $1.15(1.08$ to 1.21$)$ & 1.34 (1.05 to 1.70$)$ & 0.23 & 1.18 (1.09 to 1.29$)$ & 1.16 (1.09 to 1.25$)$ & 0.76 \\
\hline Naproxen & 1.19 (1.08 to 1.31$)$ & 0.87 (0.32 to 2.38$)$ & 0.54 & 1.24 (1.08 to 1.42$)$ & 1.15 (1.01 to 1.30$)$ & 0.43 \\
\hline Ketoprofen & 1.04 (0.95 to 1.13$)$ & 1.00 (0.50 to 2.03$)$ & 0.91 & 1.15 (1.00 to 1.32$)$ & 0.98 (0.88 to 1.09$)$ & 0.07 \\
\hline Flurbiprofen & 1.08 (0.72 to 1.62$)$ & NA & NA & 1.19 (0.62 to 2.31$)$ & 0.83 (0.50 to 1.39$)$ & 0.40 \\
\hline Oxaprozin & 0.82 (0.55 to 1.23$)$ & 0.26 (0.02 to 3.77$)$ & 0.40 & 0.45 (0.19 to 1.08$)$ & 0.96 (0.61 to 1.51) & 0.13 \\
\hline Dexibuprofen & 1.24 (0.89 to 1.74$)$ & NA & NA & 0.92 (0.50 to 1.67$)$ & 1.38 (0.93 to 2.03$)$ & 0.27 \\
\hline Celecoxib & 0.95 (0.89 to 1.02 ) & 1.05 (0.53 to 2.06$)$ & 0.77 & 1.01 (0.90 to 1.13$)$ & 0.94 (0.87 to 1.01$)$ & 0.30 \\
\hline Rofecoxib & 1.34 (1.25 to 1.44$)$ & 0.91 (0.35 to 2.42$)$ & 0.43 & 1.35 (1.20 to 1.52$)$ & $1.37(1.26$ to 1.48$)$ & 0.84 \\
\hline Valdecoxib & 1.04 (0.69 to 1.56$)$ & 0.47 (0.03 to 8.01$)$ & 0.58 & 0.95 (0.45 to 2.02) & 1.09 (0.70 to 1.71$)$ & 0.76 \\
\hline Etoricoxib & $1.55(1.42$ to 1.69$)$ & 1.35 (0.75 to 2.44$)$ & 0.65 & 1.80 (1.57 to 2.07$)$ & 1.45 (1.31 to 1.61$)$ & 0.01 \\
\hline Nabumetone & 1.07 (0.81 to 1.43$)$ & 11.14 (0.67 to 184.24$)$ & 0.10 & 1.14 (0.73 to 1.80$)$ & 1.17 (0.83 to 1.64$)$ & 0.93 \\
\hline Nimesulide & 1.21 (1.16 to 1.27$)$ & 1.00 (0.62 to 1.60$)$ & 0.43 & 1.31 (1.21 to 1.42$)$ & 1.17 (1.10 to 1.23$)$ & 0.02 \\
\hline Recent use of any NSAID & 1.01 (0.99 to 1.03$)$ & 0.97 (0.85 to 1.11$)$ & 0.55 & 1.04 (1.01 to 1.07$)$ & 0.99 (0.96 to 1.01) & 0.01 \\
\hline
\end{tabular}

glycosides, nitrates, and cytochrome P450 2C9 inhibitors). We found $9.1 \%$ of cases and $2.5 \%$ of controls with a history of heart failure diagnosis, recorded as either an outpatient diagnosis or a secondary hospital diagnosis in the year before start of NSAID treatment (cohort entry).

\section{NSAID use and heart failure risk}

A total of $16081(17.4 \%)$ cases and 1193537 (14.4\%) matched controls were current users of NSAIDs. Fig 1 reports the distribution of current use of individual NSAIDs among all cases and controls. Among controls, the most frequently used traditional NSAIDs were diclofenac $(2.9 \%)$, nimesulide $(2.4 \%)$, and ibuprofen $(1.7 \%)$, while the most frequently used COX 2 inhibitors were celecoxib (1.4\%), rofecoxib (1.0\%), and etoricoxib $(0.6 \%)$.

According to the pooled analysis, current users of any NSAID had a 20\% higher risk of heart failure than past users (odds ratio 1.19; 95\% confidence interval 1.17 to 1.22). Conversely, there was no evidence that recent use of any NSAID was associated with differences in heart failure risk with respect to past use (1.00; 0.99 to 1.02). We observed a statistically significantly higher risk of heart failure in association with current use of nine individual NSAIDs than with past use of any NSAIDs (fig 1). These NSAIDs were ketorolac, etoricoxib, indomethacin, rofecoxib, piroxicam, diclofenac, ibuprofen, nimesulide, and naproxen. Other less frequently used
NSAIDs (eg, sulindac, acemethacin, and dexibuprofen) were also found to be associated with an increased risk of heart failure, although the $95 \%$ confidence intervals included the null value. All nine significant associations identified in this analysis were also identified as significant by the Bonferroni-Holm procedure (supplementary table S2).

Compared with current use of celecoxib, current use of other individual NSAIDs was not associated with a significant decrease in heart failure risk. Odds ratios ranged from 0.83 (95\% confidence interval 0.57 to 1.20 ) for oxaprozin to 1.84 (1.67 to 2.04) for ketorolac (supplementary table S3).

For the nine individual NSAIDs significantly associated with heart failure risk, their association was also confirmed regardless of whether there was recorded evidence of a prior heart failure diagnosis and regardless of sex (table 3). The estimated risk of heart failure associated with current use of NSAIDs of nimesulide, etoricoxib, and indomethacin among women was lower in magnitude than among men, compared with past use of any NSAIDs.

According to meta-analytic analysis, current users of any NSAID had a $24 \%$ higher risk of heart failure risk than past users (odds ratio 1.24; 95\% confidence interval 1.12 to 1.36 ; fig 2 ). In addition to the nine individual NSAIDs with significant associations with heart failure risk, we found current use of nabumetone was also associated with higher risk of heart 


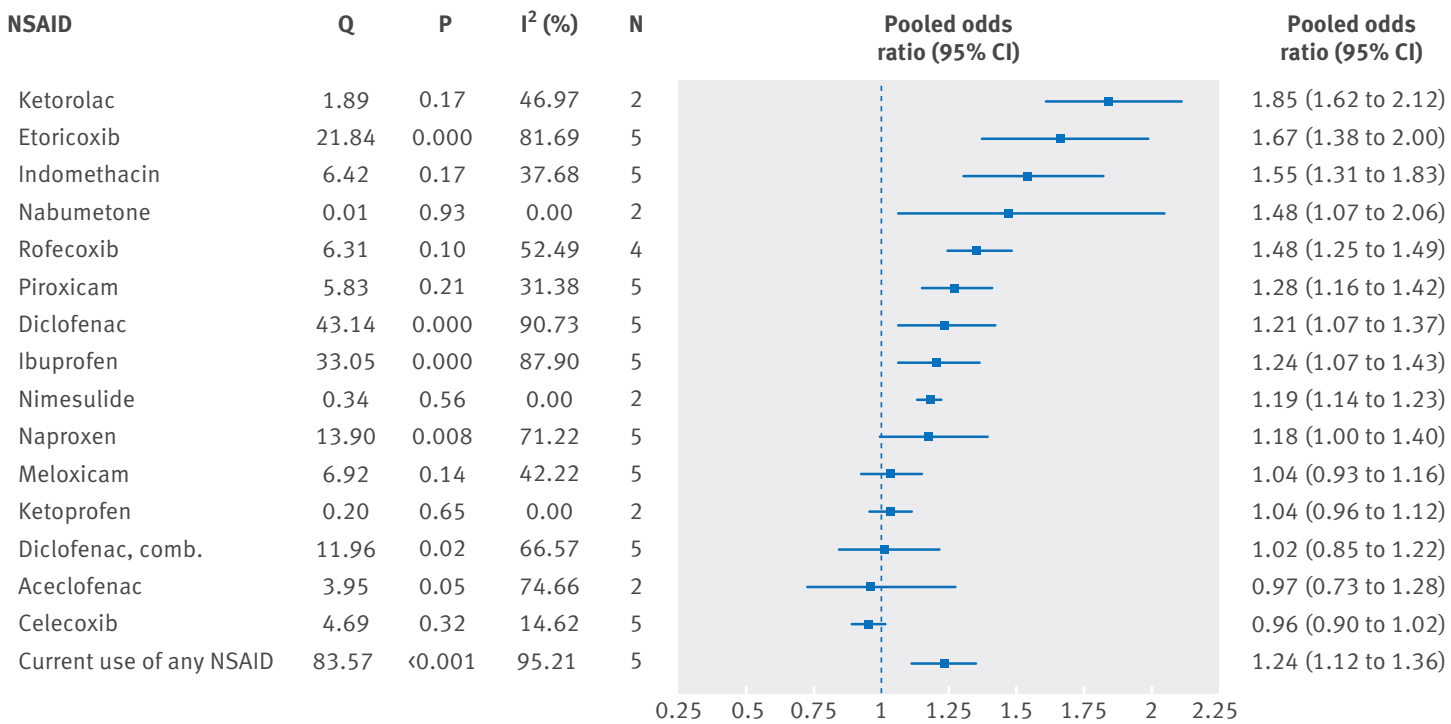

Fig 2 | Summarised associations between current use of individual NSAIDs and risk of hospital admission for heart failure, compared with past use of any NSAID. Estimates (with $95 \%$ confidence intervals) were obtained by summarising database specific odds ratios (provided at least two were available) by use of the random effects meta-analytic approach. Heterogeneity between database specific odds ratios was assessed by Cochran's Q (and corresponding P value) and Higgins' $I^{2}$ statistics. No=number of summarised databases

failure (fig 2). Although between database heterogeneity was relevant $\left(\mathrm{I}^{2}>70 \%\right)$, meta-analytic estimates of odds ratios were generally consistent with corresponding values obtained from the analysis of pooled individual level data.

\section{Dose-response relation}

Twenty $(0.2 \%)$ cases and $855(0.1 \%)$ controls from PHARMO and $753(4.3 \%)$ cases and 61777 (4.3\%) controls from THIN were excluded because prescribed daily dose data were not recorded. The remaining 25179 cases and 2083706 controls gathered from PHARMO and THIN entered the dose-response analysis.

Current users of very high doses of diclofenac, etoricoxib, indomethacin, piroxicam, and rofecoxib had more than a twofold higher risk of heart failure than past users (fig 3). The odds ratio associated with current high dose use of ibuprofen was also compatible with an increased risk of heart failure, despite the wide confidence interval. Finally, there was no evidence that celecoxib increased the risk of hospital admission heart failure at commonly used doses compared with past use of any NSAIDs. However, we cannot exclude an increase in risk when celecoxib is used at very high doses, given the wide confidence intervals obtained for this dose class.

\section{Supplementary findings}

Supplementary tables S4-S7 report the distribution of case and controls according to the considered covariates, use of individual NSAIDs, and dose categories of current NSAIDs use (in DDD equivalents and corresponding daily amount of active principle in $\mathrm{mg}$ ), as well as the effects of individual NSAIDs on the heart failure risk.

\section{Discussion}

\section{Principal findings}

Our study, based on real world data on almost 10 million NSAIDs users from four European countries, provides evidence that current use of both COX 2 inhibitors and traditional individual NSAIDs are associated with increased risk of heart failure. Furthermore, the magnitude of the association varies between individual NSAIDs and according to the prescribed dose.

NSAIDs inhibit the isoenzymes of prostaglandin $\mathrm{G} / \mathrm{H}$ synthase, COX 1 and COX $2 .{ }^{1}$ The overall effects of this inhibition of the prostaglandin synthesis are to increase peripheral systemic resistance and reduce renal perfusion, glomerular filtration rate, and sodium excretion in susceptible individuals. ${ }^{32} 33$ Taken together, these mechanisms could trigger clinical manifestations of heart failure, especially in susceptible patients. ${ }^{23}$ Additionally, because the level of prostaglandin inhibition mediated by NSAIDs increases with dose, ${ }^{14} 34$ the risk of clinical heart failure could be expected to increase along with the used NSAIDs dose.

Our study found an increased risk of hospital admission for heart failure in association with current use of several traditional NSAIDs (diclofenac, ibuprofen, indomethacin, ketorolac, naproxen, nimesulide, piroxicam, and possibly nabumetone) and two COX 2 inhibitors (etoricoxib and rofecoxib). We confirmed these findings after adjusting for multiple comparisons. Additionally, we found evidence that the increased risk of heart failure also affected patients without prior outpatient diagnosis or secondary hospital diagnosis heart failure-that is, those ideally less susceptible to heart failure decompensations. We also observed an increasing dose dependent risk of heart failure for most individual NSAIDs. Finally, indomethacin and etoricoxib 

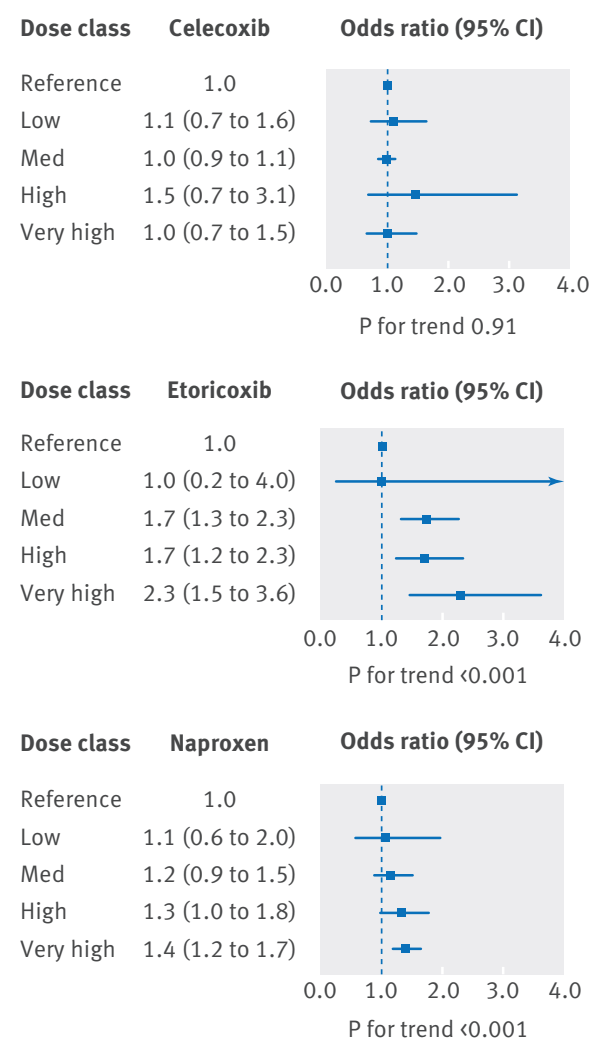
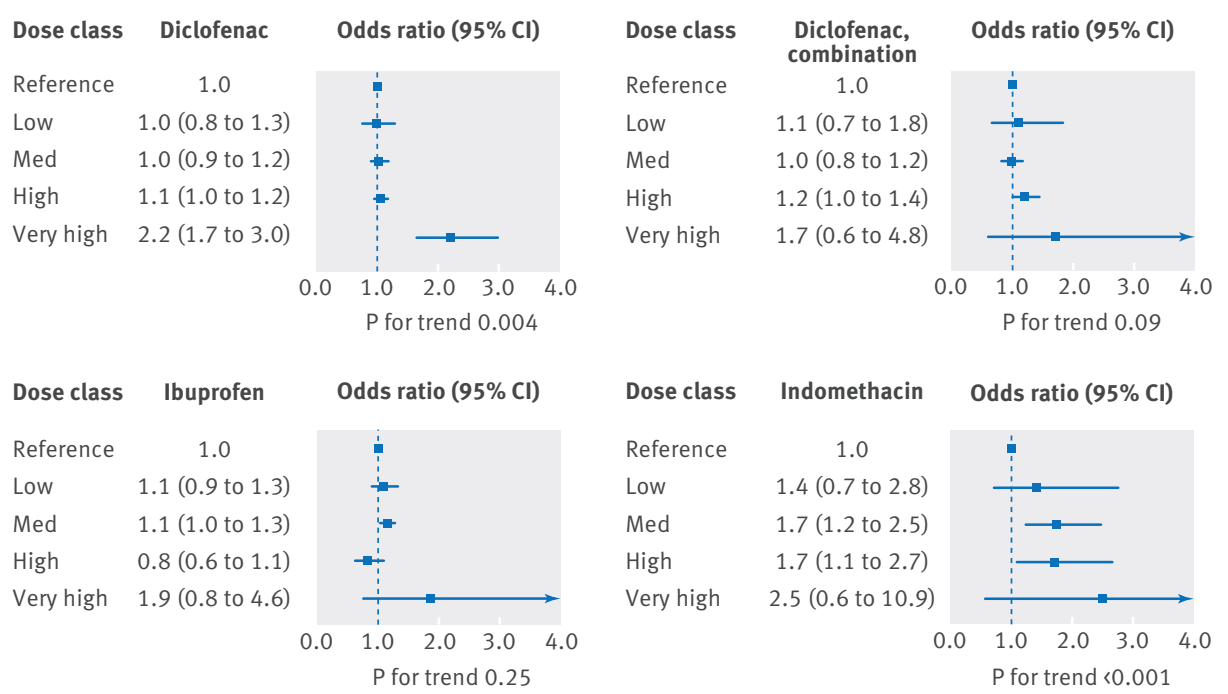

Indomethacin Odds ratio $(95 \% \mathrm{Cl})$
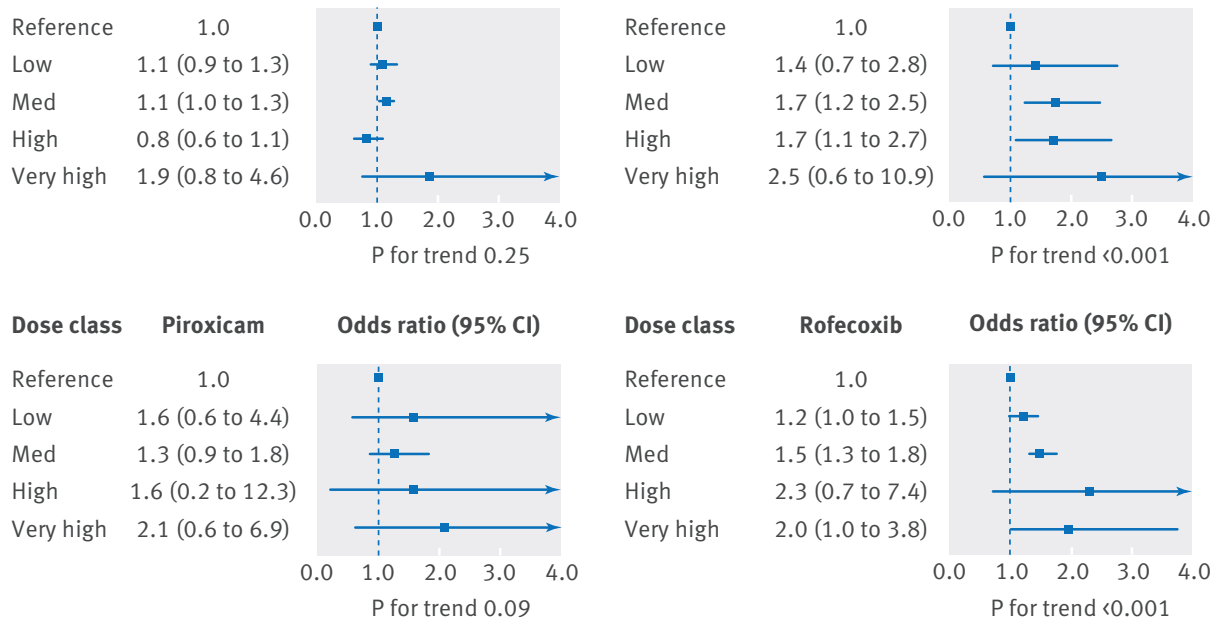

Fig 3 Dose-response relation between currently prescribed doses of specific NSAIDs and risk of heart failure, compared with past use of any NSAID. Pooled data were obtained from the Netherlands (PHARMO) and UK (THIN) databases for this analysis. Currently prescribed doses of each NSAID categorised as low (0.8 defined daily dose equivalents), medium (0.9-1.2), high (1.3-1.9), and very high ( $\geq 2)$. Odds ratios and $95 \%$ confidence intervals estimated by fitting a conditional logistic regression model after correcting for available covariates

seemed to increase the risk of hospital admission for heart failure even if used at medium doses.

No significant differences in the magnitude of the association between use of individual NSAIDs and heart failure risk were found between patients with or without prior heart failure (for all NSAIDs) and between the sexes (with a few exceptions of NSAIDs). However, power of our analysis could have been too low to detect significant differences between the considered subgroups.

Our study did not find that celecoxib, the most widely prescribed selective COX 2 inhibitor, increases the risk of hospital admission for heart failure. Lack of statistical power is unlikely explain such lack of evidence, because our main analysis had $80 \%$ power to detect significant odd ratios as low as 1.08 for the current use of celecoxib. ${ }^{35}$ Celecoxib also did not show an increased heart failure risk when used at the highest doses, although power of our analysis was low for this dose class (about 30\% power to detect significant odds ratios of 2.00). Furthermore, our study found little evidence that celecoxib is associated with a greater risk of heart failure than any of the other 26 considered individual NSAIDs.

\section{Comparison with other studies}

Our findings extend those of the meta-analysis of randomised trials, ${ }^{11}$ which showed that the risk of hospital admission for heart failure was roughly doubled by all studied NSAID regimens compared with placebo. Similarly, a meta-analysis of six trials did not show differences in heart failure risk between traditional NSAIDs and COX 2 inhibitors. ${ }^{13}$ Estimates provided by the few published observational studies on the NSAID heart failure association are compatible with an increased risk of heart failure associated with naproxen, ibuprofen, ketoprofen, piroxicam, indomethacin, and rofecoxib, but not for celecoxib. ${ }^{1423-27}$ Our results also accord with the body of evidence supporting the relative cardiovascular safety of low to medium doses of celecoxib for treatment of arthritis compared with all other COX 2 inhibitors. ${ }^{28}$

Taken together, our findings support the hypothesis that selective and non-selective COX 2 inhibitors increase the risk of heart failure, but that the magnitude of this effect varies between individual drugs and according to the dose used..$^{32}$ The effect of individual NSAIDs could depend on a complex interaction of pharmacological properties, including duration and extent of platelet inhibition, extent of blood pressure increase, and properties possibly unique to the molecule..$^{28}$

\section{Strengths and limitations of study}

Our findings, which focused only on prescription NSAIDs, might apply to NSAIDs obtained over the counter as well. Although over-the-counter NSAIDs are probably typically used at lower doses, by younger people, and for shorter durations than prescribed NSAIDs, 
they are sometimes available at the same doses than those prescribed ${ }^{36}$ and may be inappropriately overused. ${ }^{37}$ Therefore, our findings could have large scale consequences in public health and further research needs to assess the safety of over-the-counter NSAIDs under the conditions they are typically used.

The present study, conducted as part of the EU funded SOS Project, is based on data from large and unselected populations and obtained by combining different healthcare databases together. The same approach was considered in several other EU funded projects addressing various issues on drug safety, such as the arrhythmogenic risk of drugs (ARITMO project), safety of vaccines (VAESCO project), and detection of adverse drug reactions (EU ADR project). ${ }^{1819}$ The use of five different data sources from the SOS Project should be considered a strength of this study because it allowed us to compare the risk of heart failure associated with many individual NSAIDs as used in different populations and healthcare systems from four EU countries.

Our study had some limitations. Firstly, our study might not have captured all NSAID exposure, because some of these drugs (eg, ibuprofen) are also available over the counter in all the four countries. Hence, patients classified as non-current users of NSAIDs in this study might actually have been current users of over the counter NSAIDs. Such misclassification would tend to, on average, bias estimates toward the null, ${ }^{38} 39$ with the implication that our findings might understate the actual association between use of individual NSAIDs and heart failure risk.

Secondly, validity of outcome ascertainment might be of concern because heart failure is often associated with other cardiovascular diseases (eg, myocardial infarction), which could affect how hospital discharge codes are recorded. Nevertheless, although privacy concerns inhibited the validation of records in most participating databases, the positive predictive value for heart failure hospital admissions included in the Italian OSSIFF database was found to be $80 \%$ (95\% confidence interval $66 \%$ to $90 \%$ ). Additionally, high positive predictive values have been reported by other investigations based on healthcare databases for heart failure diagnosis codes at hospital discharge considered in our study. ${ }^{40}$ In fact, the incidence of almost 37.5 heart failure cases every 10000 person years observed in our study does not substantially differ from rates reported by available population based studies. ${ }^{41}$ Still, even with some outcome misclassification, ${ }^{42}$ this is expected to be non-differential-that is, independent of current use of NSAIDs-leading to a bias moving estimated associations towards the null. ${ }^{43}$ However, non-differential misclassification (of outcome or exposure) might lead to inflated observed associations due to chance alone. ${ }^{44}$

Thirdly, our dose-response analysis could have been underpowered for some NSAID dose classes because only the PHARMO and THIN databases could be considered. Additionally, a portion of patients registered in these two databases had to be excluded from the dose-response analysis because they lacked the prescribed daily dose information. Although this exclusion might have led to some bias, ${ }^{45}$ the number of excluded individuals was low and is unlikely to have had a significant effect on the results.

Fourthly, the effect of heterogeneous patient characteristics at baseline must be considered in the interpretation of our findings. Some individual NSAIDs more frequently used for different acute or chronic indications could have resulted in different patterns of use as well as in different types of populations of users. ${ }^{46}$ To address this possibility, we adjusted pooled estimates for several demographic, therapeutic, and clinical characteristics (including osteoarthritis, rheumatoid arthritis and inflammatory polyarthritis) at baseline, measured in all the included data sources. In addition, estimates did not substantially change in the random effects meta-analytic approach, where database specific estimates were adjusted for all baseline covariates available in the considered data source. Relative risk estimates for individual NSAIDs among patients with prior outpatient or secondary hospital diagnoses of heart failure (that is, those with a contraindication for NSAID use who also should be more susceptible for acute clinical manifestations of heart failure) did not seem to differ substantially from those obtained in the overall analysis. Taken together, these results provide some protection to our findings. Nevertheless, we cannot exclude that residual differences in patient's baseline characteristics could account for some of the observed variations in relative risk estimates associated with different individual NSAIDs.

Lastly, some diseases that modify both the risk of heart failure and probability of current NSAID use might not have been fully accounted for in this study. To protect against this possibility, we adjusted all our estimates for concomitant (that is, in the current period) use of specific drugs (eg, nitrates, diuretics, or other drugs for cardiovascular diseases) as a proxy of patients' current health status. Still, residual confounding cannot be excluded. For example, gout is potentially an uncontrolled confounder of the association between current use of NSAIDs and heart failure risk in this study. This is because gout is an independent risk factor for heart failure, ${ }^{47}$ and NSAIDs are the first pharmacological choice for treating acute gout episodes. ${ }^{48}$ However, the following considerations further strengthen our conclusions. We assumed that gout has a $1 \%$ prevalence in our source population and that it increases heart failure risk by 1.74 -fold. ${ }^{47} 49$ With these figures, we estimated ${ }^{50}$ that, to fully explain the observed association between naproxen and heart failure (naproxen being the NSAID with the weakest statistically significant association with heart failure in this study), acute gout episodes should have increased the odds of being treated in the current period rather than the past period by 33-fold, an implausibly high amount.

\section{Conclusions}

Our study offers further evidence that the most frequently used individual traditional NSAIDs and selective COX 2 inhibitors are associated with an increased risk of hospital admission for heart failure. Moreover, 
the risk seems to vary between drugs and according to the dose. For the individual NSAIDs less frequently used, we were not able to exclude a risk of low to moderate magnitude owing to the limited numbers of exposed cases identified in this study. Because any potential increased risk could have a considerable impact on public health, the risk effect estimates provided by this study may help inform both clinical practices and regulatory activities.

We thank all members of the Safety of Non-steroidal Anti-inflammatory Drugs (SOS) Project Consortium for their collaborative efforts.

Contributors: All authors were members of the SOS Project Consortium. AA, LS, FN, AZ, BK, TS, HS, SL, and RS were involved in data management and statistical analysis. FT, AP, and MaS were responsible for the data harmonisation procedure and data preparation. CV-L, RH, MV, VV, and SR helped to interpret the findings and manage the study. MiS was the principal investigator of the SOS Project. AA, LS, FN, AZ, and GC (guarantors) drafted the manuscript. All listed authors helped revise the final manuscript.

Funding: The research leading to the results of this study received funding from the European Community's seventh Framework Programme (grant agreement no 223495-the SOS Project). The funding source did not play any role in planning and implementing this study, in interpreting its results, and in writing this paper. Competing interests: All authors have completed the ICMJE uniform disclosure form at www.icmje.org/coi_disclosure.pdf and declare: GC collaborated with the advisory boards of Novartis and Roche and participated in projects funded by GlaxoSmithKline (GSK). HS and RH are employees of the PHARMO Institute, an independent research institute that performs financially supported studies for the government and related healthcare authorities and several pharmaceutical companies in the European Union. BK and TS work in departments that occasionally perform studies for the pharmaceutical companies, including Bayer-Schering, Celgene, GSK, Mundipharma, Novartis, Purdue, Sanofi-Aventis, Sanofi-Pasteur, Stada, and Takeda. EG runs a department that occasionally performs studies for pharmaceutical industries, including Bayer, Celgene, GSK, Mundipharma, Novartis, Sanofi, Sanofi Pasteur MSD, and STADA; has been a consultant to Bayer, Nycomed, Teva, GSK, Schwabe, and Novartis (the SOS Project was not funded or cofunded by any of these companies). SL and MV, as employees of the local health authority of Cremona, have perfomed research studies sponsored by pharmaceutical companies (Pfizer Italia, GSK, and Novartis V\&D) unrelated to this study. CV-L, as an employee of RTI Health Solutions, worked on projects funded by pharmaceutical companies including manufacturers of treatments for pain and inflammation; and participates in advisory boards funded by pharmaceutical companies. MaS has, since completion of this research, accepted a full time position at Janssen R\&D. VV, as an employee of Erasmus MC, has conducted research for AstraZeneca. MiS is head of a unit that conducts some research for pharmaceutical companies Pfizer, Novartis, Lilly, and Altana (the SOS Project was not funded or cofunded by any of these companies). All other authors have no conflicts of interest to declare. Ethical approval: Ethical approval was not required for this study. Data sharing: No additional data available.

The lead author (the manuscript's guarantor) affirms that the manuscript is an honest, accurate, and transparent account of the study being reported; that no important aspects of the study have been omitted; and that any discrepancies from the study as planned (and, if relevant, registered) have been explained.

This is an Open Access article distributed in accordance with the Creative Commons Attribution Non Commercial (CC BY-NC 3.0) license, which permits others to distribute, remix, adapt, build upon this work non-commercially, and license their derivative works on different terms, provided the original work is properly cited and the use is noncommercial. See: http://creativecommons.org/licenses/by-nc/3.0/.

1 FitzGerald GA, Patrono C. The coxibs, selective inhibitors of cyclooxygenase-2. N Engl J Med 2001;345:433-42. doi:10.1056/ NEIM200108093450607

2 Fries JF. Selective cyclooxygenase inhibition: promise for future NSAID therapy?Scand J Rheumatol Suppl 1996;102:1. doi:10.3109/03009749609097224.

3 Bombardier C, Laine L, Reicin A, et al. VIGOR Study Group. Comparison of upper gastrointestinal toxicity of rofecoxib and naproxen in patients with rheumatoid arthritis. N Engl J Med 2000;343:1520-8, 2, 1528. doi:10.1056/NEJM200011233432103.
4 Amer M, Bead VR, Bathon J, Blumenthal RS, Edwards DN. Use of nonsteroidal anti-inflammatory drugs in patients with cardiovascular disease: a cautionary tale Cardiol Rev 2010;18:204-12. doi:10.1097/ CRD.0b013e3181ce1521.

5 Bresalier RS, Sandler RS, Quan H, et al. Adenomatous Polyp Prevention on Vioxx (APPROVe) Trial Investigators. Cardiovascular events associated with rofecoxib in a colorectal adenoma chemoprevention trial. N Engl I Med 2005;352:1092-102. doi:10.1056/NEJMoa050493.

6 Solomon SD, McMurray JJ, Pfeffer MA, et al. Adenoma Prevention with Celecoxib (APC) Study Investigators. Cardiovascular risk associated with celecoxib in a clinical trial for colorectal adenoma prevention. N Engl I Med 2005;352:1071-80. doi:10.1056/NEJMoa050405.

7 Kearney PM, Baigent C, Godwin J, Halls H, Emberson JR, Patrono C. Do selective cyclo-oxygenase-2 inhibitors and traditional non-steroidal anti-inflammatory drugs increase the risk of atherothrombosis? Meta-analysis of randomised trials. BM/ 2006;332:1302-8. doi:10.1136/bmj.332.7553.1302

8 García Rodríguez LA, Tacconelli S, Patrignani P. Role of dose potency in the prediction of risk of myocardial infarction associated with nonsteroidal anti-inflammatory drugs in the general population. J Am Coll Cardiol 2008;52:1628-36. doi:10.1016/j.jacc.2008.08.041.

9 McGettigan P. Henry D. Cardiovascular risk with non-steroidal anti-inflammatory drugs: systematic review of population-based controlled observational studies. PLoS Med 2011;8:e1001098. doi:10.1371/journal.pmed.1001098.

10 Trelle S, Reichenbach S, Wandel S, et al. Cardiovascular safety of non-steroidal anti-inflammatory drugs: network meta-analysis. BMJ 2011;342:c7086. doi:10.1136/bmj.c7086.

11 Bhala N, Emberson J, Merhi A, et al. Coxib and traditional NSAID Trialists' (CNT) Collaboration. Vascular and upper gastrointestinal effects of non-steroidal anti-inflammatory drugs: meta-analyses of individual participant data from randomised trials. Lancet 2013;382:769-79. doi:10.1016/S0140-6736(13)60900-9.

12 Fabule J, Adebajo A. Comparative evaluation of cardiovascular outcomes in patients with osteoarthritis and rheumatoid arthritis on recommended doses of nonsteroidal anti-inflammatory drugs. Ther Adv Musculoskelet Dis 2014;6:111-30. doi:10.1177/1759720X14541668.

13 Scott PA, Kingsley GH, Scott DL. Non-steroidal anti-inflammatory drugs and cardiac failure: meta-analyses of observational studies and randomised controlled trials. Eur J Heart Fail 2008;10:1102-7. doi:10.1016/j.ejheart.2008.07.013.

14 García Rodríguez LA, Hernández-Díaz S. Nonsteroidal antiinflammatory drugs as a trigger of clinical heart failure. Epidemiology 2003;14:2406. doi:10.1097/01.EDE.0000034633.74133.C3.

15 McMurray JJ, Adamopoulos S, Anker SD, et al. ESC Committee for Practice Guidelines. ESC Guidelines for the diagnosis and treatment of acute and chronic heart failure 2012: The Task Force for the Diagnosis and Treatment of Acute and Chronic Heart Failure 2012 of the European Society of Cardiology. Developed in collaboration with the Heart Failure Association (HFA) of the ESC. Eur Heart / 2012;33:1787847. doi:10.1093/eurheartj/ehs104.

16 Ray WA. Evaluating medication effects outside of clinical trials: new-user designs. Am J Epidemiol 2003;158:915-20. doi:10.1093/ aje/kwg231.

17 Valkhoff VE, Schade R, 't Jong GW, et al. Safety of Non-steroidal Anti-inflammatory Drugs (SOS) project. Population-based analysis of non-steroidal anti-inflammatory drug use among children in four European countries in the SOS project: what size of data platforms and which study designs do we need to assess safety issues?BMC Pediatr 2013;13:192. doi:10.1186/1471-2431-13-192.

18 Avillach P, Coloma PM, Gini R, et al. EU-ADR consortium Harmonization process for the identification of medical events in eight European healthcare databases: the experience from the EU-ADR project. / Am Med Inform Assoc 2013;20:184-92. doi:10.1136/amiajnl-2012-000933.

19 Trifirò G, Coloma PM, Rijnbeek PR, et al. Combining multiple healthcare databases for postmarketing drug and vaccine safety surveillance: why and how? J Intern Med 2014;275:551-61. doi:10.1111/joim.12159.

20 Hosmer DW, Lemeshow S. Applied Logistic Regression.2nd ed. John Wiley \& Sons, 2000. doi:10.1002/0471722146.

21 Wright SP. Adjusted p-values for simultaneous inference. Biometrics 1992;48:1005-13. doi:10.2307/2532694

22 Sedgwick P. Pitfalls of statistical hypothesis testing: multiple testing [correction in: BMJ 2014;349:g5624]. BMJ 2014;349:g5310. doi:10.1136/bmj.g5310.

23 Huerta C, Varas-Lorenzo C, Castellsague J, García Rodríguez LA Non-steroidal anti-inflammatory drugs and risk of first hospital admission for heart failure in the general population. Heart 2006;92:1610-5. doi:10.1136/hrt.2005.082388.

24 Bernatsky S, Hudson M. Suissa S. Anti-rheumatic drug use and risk of hospitalization for congestive heart failure in rheumatoid arthritis. Rheumatology (Oxford) 2005;44:677-80. doi:10.1093/rheumatology/ keh610. 
25 Mamdani M, Juurlink DN, Lee DS, et al. Cyclo-oxygenase-2 inhibitors versus non-selective non-steroidal anti-inflammatory drugs and congestive heart failure outcomes in elderly patients: a populationbased cohort study. Lancet 2004;363:1751-6. doi:10.1016/ S0140-6736(04)16299-5.

26 Mangoni AA, Woodman RJ, Gaganis P, Gilbert AL, Knights KM. Use of non-steroidal anti-inflammatory drugs and risk of incident myocardial infarction and heart failure, and all-cause mortality in the Australian veteran community. BrJ Clin Pharmacol 2010;69:689-700. doi:10.1111/j.1365-2125.2010.03627.x

27 McGettigan P, Han P, Jones L, Whitaker D, Henry D. Selective COX-2 inhibitors, NSAIDs and congestive heart failure: differences between new and recurrent cases. Br J Clin Pharmacol 2008;65:927-34. doi:10.1111/j.1365-2125.2008.03121.x.

28 Howes LG. Selective COX-2 inhibitors, NSAIDs and cardiovascular events - is celecoxib the safest choice? Ther Clin Risk Manag 2007:3:831-45

29 Cooper H, Patall EA. The relative benefits of meta-analysis conducted with individual participant data versus aggregated data. Psychol Methods 2009:14:165-76. doi:10.1037/a0015565.

30 DerSimonian R, Laird N. Meta-analysis in clinical trials. Control Clin Trials 1986;7:177-88. doi:10.1016/0197-2456(86)90046-2.

31 Higgins JP, Thompson SG. Quantifying heterogeneity in a metaanalysis. Stat Med 2002;21:1539-58. doi:10.1002/sim.1186.

32 Patrono C, Baigent C. Nonsteroidal anti-inflammatory drugs and the heart. Circulation 2014;129:907-16. doi:10.1161/ CIRCULATIONAHA.113.004480.

33 Bleumink GS, Feenstra J, Sturkenboom MC, Stricker BH. Nonsteroida anti-inflammatory drugs and heart failure. Drugs 2003;63:525-34. doi:10.2165/00003495-200363060-00001.

34 Bäck M, Yin L, Ingelsson E. Cyclooxygenase-2 inhibitors and cardiovascular risk in a nation-wide cohort study after the withdrawal of rofecoxib. Eur Heart / 2012;33:1928-33. doi:10.1093/eurheartj/ehr421.

35 Lui KJ. Estimation of sample sizes in case-control studies with multiple controls per case: dichotomous data. Am J Epidemio 1988; 127:1064-70

36 Duong M, Salvo F, Pariente A, et al. Usage patterns of 'over-the-counter' vs. prescription-strength nonsteroidal anti-inflammatory drugs in France. BrJ Clin Pharmacol 2014;77:887-95. doi:10.1111/bcp.12239.

37 Koffeman AR, Valkhoff VE, Celik S, et al. High-risk use of over-thecounter non-steroidal anti-inflammatory drugs: a population-based cross-sectional study. Br J Gen Pract 2014;64:e191-8. doi:10.3399/ bjgp14X677815.

38 Yood MU Campbell UB, Rothman KJ, et al. Using prescription claims data for drugs available over-the-counter (OTC). Pharmacoepidemiol Drug Saf 2007;16:961-8. doi:10.1002/pds.1454.
39 Delaney JA, Biggs ML, Kronmal RA, Psaty BM. Demographic, medical, and behavioral characteristics associated with over the counter non-steroidal anti-inflammatory drug use in a population-based cohort: results from the Multi-Ethnic Study of Atherosclerosis. Pharmacoepidemiol Drug Saf 2011;20:83-9. doi:10.1002/pds.2065.

40 Saczynski IS, Andrade SE, Harrold LR, et al. A systematic review of validated methods for identifying heart failure using administrative data. Pharmacoepidemiol Drug Saf 2012;21(Suppl 1):129-40. doi:10.1002/pds.2313.

41 Corrao G, Ghirardi A, Ibrahim B, Merlino L, Maggioni AP. Burden of new hospitalization for heart failure: a population-based investigation from Italy. Eur J Heart Fail 2014;16:729-36. doi:10.1002/ejhf.105.

42 Romano PS, Mark DH. Bias in the coding of hospital discharge data and its implications for quality assessment. Med Care 1994;32:81-90. doi:10.1097/00005650-199401000-00006.

43 Copeland KT, Checkoway H, McMichael AJ, Holbrook RH. Bias due to misclassification in the estimation of relative risk. Am J Epidemio 1977;105:488-95.

44 Jurek AM, Greenland S, Maldonado G, Church TR. Proper interpretation of non-differential misclassification effects: expectations vs observations. Int J Epidemiol 2005;34:680-7. doi:10.1093/ije/dyi060.

45 Altman DG, Bland JM. Missing data. BMJ 2007;334:424. doi:10.1136/ bmj.38977.682025.2C

46 Moride Y, Ducruet T, Boivin JF, Moore N, Perreault S, Zhao S Prescription channeling of COX-2 inhibitors and traditional nonselective nonsteroidal anti-inflammatory drugs: a populationbased case-control study. Arthritis Res Ther 2005;7:R333-42. doi:10.1186/ar1488

47 Krishnan E. Gout and the risk for incident heart failure and systolic dysfunction. BMJ Open 2012;2:e000282. doi:10.1136/ bmjopen-2011-000282.

48 Kim KY, Ralph Schumacher H, Hunsche E, Wertheimer Al, Kong SX. A literature review of the epidemiology and treatment of acute gout. Clin Ther 2003;25:1593-617. doi:10.1016/S0149-2918(03)80158-3.

49 Smith EU, Díaz-Torné C, Perez-Ruiz F, March LM. Epidemiology of gout: an update. Best Pract Res Clin Rheumatol 2010;24:811-27. doi:10.1016/j.berh.2010.10.004

50 Schneeweiss S. Sensitivity analysis and external adjustment for unmeasured confounders in epidemiologic database studies of therapeutics. Pharmacoepidemiol Drug Saf 2006;15:291-303. doi:10.1002/pds.1200

Supplementary appendix: Additional material 